\title{
RESEARCH
}

Open Access

\section{Participant and study partner prediction and identification of cognitive impairment in preclinical Alzheimer's disease: study partner vs. participant accuracy}

Mary M. Ryan ${ }^{1 *}$, Joshua D. Grill ${ }^{2,3,4,5^{*+}}$ D , Daniel L. Gillen ${ }^{1,2^{*}+}$ and for the Alzheimer's Disease Neuroimaging Initiative

\begin{abstract}
Background: Preclinical Alzheimer's disease (AD) clinical trials require participants to enroll with a study partner, a person who can attend visits and report changes in the participant's cognitive ability. Whether study partners, compared to participants themselves, provide added information about participant cognition in preclinical AD trials is an open question. We tested the hypothesis that study partners provide meaningful information related to participant cognition cross-sectionally and longitudinally, and assessed whether amyloid status modified observed effects.
\end{abstract}

Methods: We assessed participant and study partner Everyday Cognition (ECog) scores and participant Alzheimer's Disease Assessment Scale 13-item cognitive subscale (ADAS13) data from 335 cognitively normal participant-partner dyads in the AD Neuroimaging Initiative. We used random forest and linear mixed effects (LME) models to predict ADAS13 scores as a function of participant and/or study partner ECog scores over time. LME models were adjusted for potential confounding factors, including APOE4 status, amyloid status, baseline age, years of education, and sex. Random forest models were split into the above factors, as well as race/ethnicity and other available neuropsychological battery test scores.

Results: In random forest models predicting ADAS13 12 months from baseline, we observed no difference in the estimated mean variable importance (eMVI) associated with baseline study partner ECog compared to the baseline participant ECog (eMVI = 0.15, 95\%CB 0.13, 0.16 for partner; $\mathrm{eMVI}=0.15,95 \% \mathrm{CB} 0.14,0.16$ for participant). In models predicting ADAS13 48 months after baseline, the eMVI associated with baseline study partner ECog was slightly lower than that associated with baseline participant ECog (eMVI =0.21, 95\%CB 0.20, 0.22 for partner; eMVI $=0.24$, 95\%CB 0.22, 0.25 for participant). In cross-sectional models, study partner eMVI was twice as large as participant eMVI at 12 months (eMVI $=0.20,95 \%$ CB 0.19, 0.21 for partner; eMVI $=0.09,95 \% C B$ 0.09, 0.10 for participant) and three times as large at 48 months (eMVI $=0.38,95 \%$ CB 0.36, 0.39 for partner; eMVI $=0.13,95 \% C B \quad 0.12,0.14$ for participant). We did not observe qualitative differences by amyloid status.

Conclusions: While baseline participant reports reasonably predict subsequent cognitive change, informants perform better at cross-sectionally recognizing cognitive status as observation time grows. The study partner requirement may be essential to ensure trial data integrity, especially in longer trials.

Keywords: Alzheimer's disease, Study partner, preclinical, ADNI

\footnotetext{
*Correspondence: marymr@uci.edu; jgrill@uci.edu; dgillen@uci.edu

†Joshua D. Grill and Daniel L. Gillen are co-senior authors.

'Department of Statistics, University of California, Irvine, Irvine, CA 92697, USA

${ }^{2}$ Institute for Memory Impairments and Neurological Disorders, University of

California, Irvine 92697, CA, USA

Full list of author information is available at the end of the article
}

(c) The Author(s). 2019 Open Access This article is distributed under the terms of the Creative Commons Attribution 4.0 International License (http://creativecommons.org/licenses/by/4.0/), which permits unrestricted use, distribution, and

reproduction in any medium, provided you give appropriate credit to the original author(s) and the source, provide a link to the Creative Commons license, and indicate if changes were made. The Creative Commons Public Domain Dedication waiver (http://creativecommons.org/publicdomain/zero/1.0/) applies to the data made available in this article, unless otherwise stated. 


\section{Introduction}

Alzheimer's disease (AD) is a progressive neurodegenerative disease that results in dementia-cognitive and functional impairment that interrupts independence in daily life. US Food and Drug Administration (FDA) approval for new AD treatments historically requires demonstration of both functional and cognitive benefit in AD dementia clinical trials. Demonstration of functional benefit, along with logistical needs related to study compliance and ethical needs related to informed consent, requires that $\mathrm{AD}$ dementia trial participants enroll with a study partner $[1,2]$.

In an effort to intervene earlier in the disease process, before neurodegeneration reaches severe stages, researchers have begun performing clinical trials that enroll patients whose disease does not meet the criteria for dementia. This includes trials of patients with mild cognitive impairment (MCI), a construct defined by objective cognitive impairment without functional loss or impaired activities of daily living. MCI patients with biomarker evidence of $\mathrm{AD}$, such as elevated brain levels of amyloid- $\beta_{42}$, which can be observed through the use of neuroimaging and cerebrospinal fluid (CSF) protein analysis [3], meet the criteria for $M C I$ due to $A D$ or prodromal $A D[4,5]$. To intervene even earlier in disease, preclinical $A D$ trials enroll patients with no cognitive impairment but biomarker evidence of $\mathrm{AD}$ [6].

Participants in preclinical and prodromal AD trials are expected to be able to provide informed consent and to comply with study requirements. Yet, the need for informants who report participant cognitive and functional performance in these trials is less understood. Study partners may perform better at predicting future AD dementia than do patients with MCI [7, 8], while other studies show that patients with MCI are fairly accurate at assessing their current cognitive state [9]. Preliminary studies indicate that self-reports from cognitively normal participants may better predict future outcomes than do study partners $[10,11]$. Whether these relationships are altered in the presence of AD biomarkers remains an area in need of study.

Initial preclinical $\mathrm{AD}$ trials require participants to enroll with study partners [12]. An open question remains, though, whether participants themselves or their partners provide more meaningful information on trial outcomes. In this study, we sought to determine if study partners provide additional information, in relation to preclinical $\mathrm{AD}$ study participants, in predicting future cognitive decline or assessing current cognitive performance, and assessed whether amyloid status modified observed effects. We hypothesized that study partners would provide more meaningful information than participants over time, but that this effect would be observed only in participants with elevated brain amyloid.

\section{Methods}

\section{Data collection}

We used data from the AD Neuroimaging Initiative (ADNI). Data used in the preparation of this article were obtained from the ADNI database (adni.loni.usc.edu). The ADNI was launched in 2003 as a public-private partnership, led by principal investigator Michael W. Weiner, MD. The primary goal of ADNI has been to test whether serial magnetic resonance imaging (MRI), positron emission tomography (PET), other biological markers, and clinical and neuropsychological assessment can be combined to measure the progression of MCI and early AD. For up-to-date information, see www. adni-info.org.

Criteria for this study were that participants have a diagnosis of "cognitively normal" at the first visit with an available Everyday Cognition (ECog) score and have at least one observation of amyloid status, as observed via CSF measurement, PET AV45 standard uptake value ratio (SUVR), or PET Pittsburgh compound B (PiB). We used the first visit in which there was an available ECog score as the baseline for this study. After implementing this inclusion/exclusion criteria, there were $N=335$ viable dyads for the current study: $N=227$ had data available at a visit 12 months after baseline, $N=250$ at 24 months, and $N=107$ at 48 months.

On May 9, 2014, an ADNI2 protocol amendment restricted annual ADNI visits to those participants who progressed to $\mathrm{MCI}$ or dementia; cognitively normal participants completed visits every 2 years [13]. The change occurred after all ADNI2 participants were assessed for their 12 -month visit, so only month 36 visits were affected. While the protocol change did not impact the entire available data pool, it was significant enough that observed results at 36 months would potentially be biased. Therefore, we excluded any observations collected at 36 months from the current analysis.

\section{Amyloid positivity}

We assigned amyloid status based on CSF amyloid beta $\left(A \beta_{42}\right)$, AV45 SUVR, or PiB measurements. PiB measurements were converted to the AV45 SUVR scale via the regression equation $y=0.15+0.67 x$ [14]. Those participants with either CSF $A \beta_{42}$ levels below $192 \mathrm{pg} / \mathrm{mL}$ or AV45 SUVR (converted or otherwise) above 1.1 were classified as "elevated amyloid": all others were designated "not elevated amyloid."

\section{Alzheimer's Disease Assessment Scale 13-item cognitive subscale (ADAS13)}

We used the Alzheimer Disease Assessment Scale 13item cognitive subscale (ADAS13) as an objective cognitive performance response variable in this study. Compared to the 11-item version used in dementia trials, the 
ADAS13 includes a number of cancelation and a delayed free recall task that increases sensitivity in early-stage disease $[15,16]$. The ADAS13 has a range of 85 possible points, with higher scores reflecting poorer cognitive performance.

\section{Everyday Cognition}

We used the Everyday Cognition (ECog) scale to examine the subjective cognitive performance in this study. The ECog includes both participant and informant versions. The ECog is a 39-item questionnaire designed to measure functional performance that is linked to specific cognitive abilities [17]. It compares the participant's current everyday functioning with their perceived functioning (or their study partner's perception of their functioning) from 10 years prior. Each item covers a task in 1 of 6 cognitively relevant domains (memory, language, visuospatial abilities, planning, organization, and divided attention) and is rated on a 4-point scale, with 1 being "better or no change compared with 10 years earlier" and 4 being "consistently much worse." Overall ECog scores are calculated by averaging the ratings from the 39 items.

\section{Statistical methods}

Study population demographics were summarized via sample means and standard deviations for continuous variables, and counts and percentages for discrete variables.

We sought to quantify the ability of study participants and partners to cross-sectionally predict cognitive performance and to prospectively predict future changes in cognition, as measured by the ADAS13. We used random forest models to assess the predictive ability of participants and study partners over the course of observation, modeling ADAS13 scores at 12, 24, and 48 months as a function of baseline participant and/or study partner ECog scores [18]. Random forest models represent an ensemble (or average) of Classification and Regression Trees constructed via recursive partitioning where binary separations of the study sample are created by choosing the optimal predictor and cut-point combination to yield the largest discrimination in mean response values between the resulting subpopulations. Random forest models were trained using crossvalidation with squared error loss as the prediction penalty, as implemented in the $\mathrm{R}$ statistical software language using the GRF package $[19,20]$. To account for potential imbalance in the number of repeated measures across subjects, we fit models using a multiple outputation procedure in which a balanced number of observations were sampled from each subject [21]. To maximize efficiency, we took the number of randomly sampled observations per subject to be the minimum number of repeated measures on any one participant in the study sample.

We calculated estimated mean variable importance (eMVI) to assess the relative informative and predictive abilities of participant and study partner ECog scores in the random forest models. Variable importance is measured here as the weighted sum of the frequencies at which a variable is used to split the dataset at various levels-when a variable is used to split the dataset at the top of the decision tree, it is given more weight than when it is used to split the dataset farther down the tree. The larger the variable importance measure, the more important the variable is within the model. To account for stochasticity in the formulation of the random forest models, we created 100 forests by varying the random seed generator in $\mathrm{R}$ and calculated the eMVI by taking the sample average of the variable importance measures from each forest. We calculated 95\% error bounds by taking the 2.5 and 97.5th percentiles of the simulated variable importance measures.

To provide additional interpretability of the impact of the participant and partner subjective assessment on recognizing participant cognitive decline, we also built linear mixed effects (LME) models on a reduced set of covariates, chosen a priori. As with the random forest models, we modeled ADAS13 scores at 12, 24, and 48 months as a function of baseline participant and/or study partner ECog scores. Specifically, we considered models of the form:

$$
\operatorname{ADAS}_{1, j}=\vec{X}_{i, 0} \vec{\beta}+\gamma_{i},
$$

where $\mathrm{ADAS1}_{i, j}$ represents the ADAS13 participant score for participant $i$ at time point $j ; \vec{X}_{i, 0}$ denotes the vector of baseline covariates for subject $i$, including participant and/or study partner ECog score; $\vec{\beta}$ represents a vector of fixed effects parameters associated with the baseline covariates; and $\gamma_{i}$ denotes a subject-specific random intercept. In addition, all models were adjusted for potential confounding factors, including APOE4 status (carrier vs. non-carrier), amyloid status (elevated vs. not elevated), baseline age, years of education, and sex. Residual diagnostics were conducted to assess the assumption of the exchangeable covariance structure implied by the random intercept model.

To assess the informative ability of participants and study partners at a given time point, we created crosssectional random forest and LME models using ECog scores at 12, 24, and 48 months to predict ADAS13 scores at the same time points. We used a similar approach as above, except baseline ECog scores were replaced with concurrent scores for the visit at which the response was obtained. 


\section{Results}

Table 1 describes the demographics of the participants included in this analysis, stratified by amyloid status. Compared to the not elevated group, the elevated amyloid group included more female participants $(77 \%$ vs. $47 \%$ ) and more participants with at least one $\varepsilon 4$ allele of the APOE gene ( $47 \%$ vs. $23 \%$ ). Both groups had similar mean ages, years of education, and participant and study partner baseline ECog scores, though the elevated amyloid group had higher baseline ADAS13 scores (9.63 vs. 8.65). In both the elevated and the not elevated amyloid groups, participants scored their cognitive function on the ECog worse than did their study partners at baseline. Demographic data for ADNI study partners were not available.

In random forest models predicting ADAS13 12 months from baseline, the eMVI associated with baseline study partner ECog was not different from that associated with baseline participant ECog $(e M V I=0.15$, $95 \% \mathrm{CB} 0.13,0.16$ for partner; $\mathrm{eMVI}=0.15,95 \% \mathrm{CB} 0.14$, 0.16 for participant; Fig. 1a). Similarly, when predicting ADAS13 further from baseline to 48 months, the eMVI associated with baseline study partner ECog was slightly lower than that associated with baseline participant ECog $(\mathrm{eMVI}=0.21,95 \% \mathrm{CB} \quad 0.20,0.22$ for partner; eMVI $=0.24,95 \% \mathrm{CB} 0.22,0.25$ for participant; Fig. 1a). These results were mirrored in the longitudinal LME models predicting the same time points (see Tables 2 and 3 ).
In cross-sectional random forest models assessing 12month ADAS13, the eMVI associated with study partner ECog at 12 months was twice as large as the eMVI associated with participant ECog at 12 months (eMVI $=0.20$, 95\%CB 0.19, 0.21 for partner; eMVI = 0.09 95\%CB 0.09, 0.10 for participant; Fig. 1b). By 48 months, the eMVI associated with study partner ECog for assessing ADAS13 was three times as large as that associated with participant ECog-a statistically significant difference $(\mathrm{eMVI}=$ $0.38,95 \% \mathrm{CB} 0.36,0.39$ for partner; $\mathrm{eMVI}=0.13,95 \% \mathrm{CB}$ 0.12, 0.14 for participant; Fig. 1b).

A similar gap was observed at 48 months in the crosssectional LME model: a 1 standard deviation increase in study partner ECog was associated with a 1.37-point increase in ADAS13 score (95\% CI 0.78, 1.96; Table 3) while a 1 standard deviation increase in participant ECog was associated with a 0.66 -point increase $(95 \% \mathrm{CI}-0.17$, 1.48; Table 3). Removing cases of conversion to MCI from the analyses had minimal impact at 12 and 24 months but essentially removed the observed difference in eMVI between partners and participants at 48 months (data not shown).

Across all LME models-including those predicting future ADAS13 scores from baseline ECog scores and cross-sectional models-the effect of amyloid status was not statistically significant. Likewise, there were there no significant interacting effects between amyloid status and participant or study partner ECog scores in any LME model.

Table 1 Characteristics of participants and study partners analyzed

\begin{tabular}{llll}
\hline & Amyloid beta elevated $(n=100)$ & Amyloid beta not elevated $(n=235)$ & Total $(n=335)$ \\
\hline Sex & & & \\
$\quad$ Male & $33(33 \%)$ & $124(52.77 \%)$ & $157(46.87 \%)$ \\
$\quad$ Female & $77(77 \%)$ & $111(47.23 \%)$ & $178(53.13 \%)$ \\
Race/ethnicity & & & $301(89.85 \%)$ \\
$\quad$ Caucasian & $89(89 \%)$ & $212(90.21 \%)$ & $22(6.57 \%)$ \\
$\quad$ African-American & $7(7 \%)$ & $15(6.38 \%)$ & $6(1.79 \%)$ \\
Asian & $2(2 \%)$ & $4(1.7 \%)$ & $73.26(5.83)$ \\
Age & $74.34(5.87)$ & $72.8(5.77)$ & $16.49(2.58)$ \\
Years of education & $15.93(2.56)$ & $16.72(2.55)$ & \\
APOE4 alleles & & $182(77.45 \%)$ & $235(70.15 \%)$ \\
$\quad 0$ & $53(53 \%)$ & $49(20.85 \%)$ & $92(27.46 \%)$ \\
1 & $43(43 \%)$ & $4(1.70 \%)$ & $8(2.39 \%)$ \\
2 & $4(4 \%)$ & $29.08(1.28)$ & $29.07(1.22)$ \\
MMSE & $29.04(1.08)$ & $8.65(4.42)$ & $8.94(4.44)$ \\
ADAS13 & $9.63(4.43)$ & $1.38(0.33)$ & $1.20(0.30)$ \\
ECog & $1.42(0.32)$ & & $1.39(0.33)$ \\
Participant & $1.21(0.29)$ & $0.29)$ & 1.20 \\
\hline Study partner & & & \\
\hline
\end{tabular}




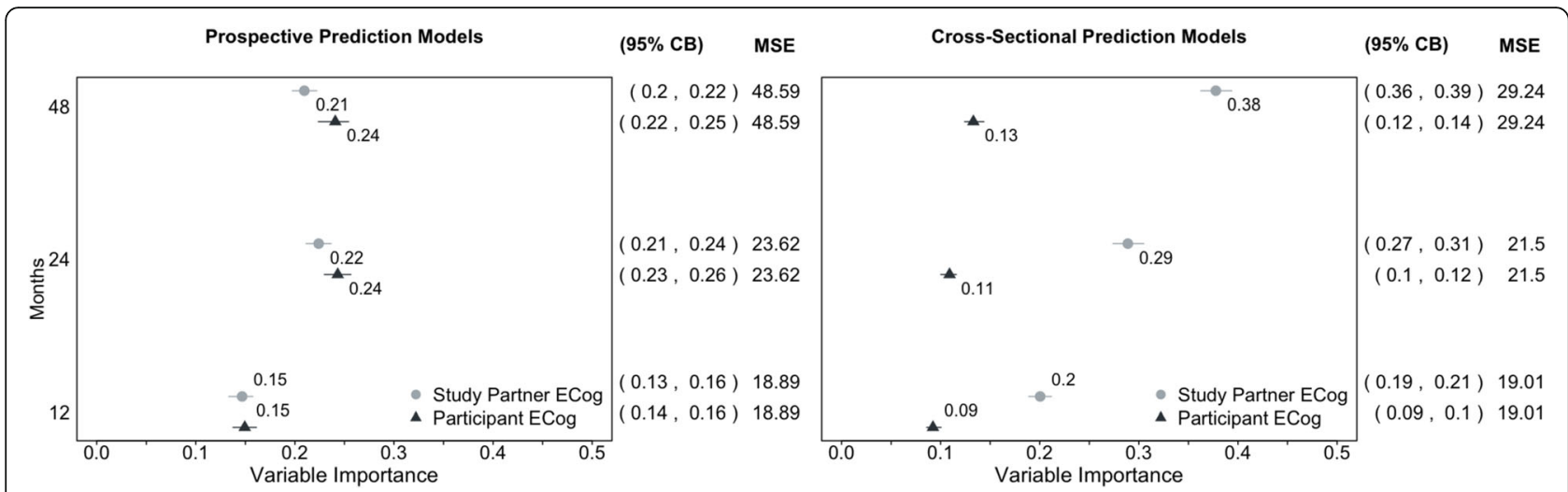

Fig. 1 Variable importance of baseline (a) and 12-, 24-, and 48-month (b) participant and study partner ECog scores predicting 12-, 24-, and 48month ADAS13 scores in the additive model using the same covariates as in the LME models

\section{Discussion}

We investigated the extent to which study partners provide relevant additional information, in relation to study participants, in a sample that parallels preclinical AD trials. While eMVI values cannot be compared across models, because the outcomes measured in each model are different and hence yield inherently different variation, we can compare the relative performance of participants and study partners within each setting. When we assessed the participants' and partners' abilities at baseline to predict future cognitive performance through random forest models, we found no difference between the groups. When examining the relationships between participant and partner reporting cross-sectionally, however, we found that the study partners provide increasingly meaningful information over time and that this information eventually (by 48 months) becomes more valuable than the participant's self-report. Similar results were seen in the associative LME models: higher study partner ECog scores were associated with higher ADAS13 scores, compared to ECog scores provided by participants, in both longitudinal and crosssectional models. In the prospective prediction case, the width of this gap stayed approximately static over time, whereas in cross-sectional models, this gap widened over time.

These results are similar to the previous observations and indicate that the information provided by the study partners becomes increasingly important over the course of a preclinical AD trial. For example, Amariglio and colleagues observed higher correlations between participant Cognitive Function Instrument (CFI) scores and a composite cognitive outcome at baseline, compared to study partner CFI scores. By 48 months, however, correlations were higher for partners compared to participants, though summing CFI for the dyad demonstrated the highest correlations [11]. Two, not mutually exclusive, potential explanations exist for why study partners may increasingly outperform participants in recognizing cognitive impairment in preclinical AD trials. First, the quality of the information provided by the study partner may increase with time, as they become more comfortable with the instrument, study procedures, and the need to observe participant behaviors between visits. Second, some participants may experience cognitive decline over the course of the study, rendering them less able to reliably report their own cognitive and functional

Table 2 LME coefficients for longitudinal and cross-sectional models at 12 months from baseline

\begin{tabular}{|c|c|c|c|c|}
\hline & \multicolumn{2}{|l|}{ Longitudinal model } & \multicolumn{2}{|l|}{ Cross-sectional model } \\
\hline & Coefficient estimate & 95\% confidence interval & Coefficient estimate & $95 \%$ confidence interval \\
\hline Participant ECog & 0.16 & $(-0.50,0.79)$ & 0.06 & $(-0.44,0.56)$ \\
\hline Study partner ECog & 0.72 & $(0.07,1.36)$ & 0.67 & $(0.11,1.23)$ \\
\hline Time & -1.22 & $(-184.30,181.86)$ & -14.02 & $(-200.75,172.71)$ \\
\hline Squared time & 3.17 & $(-89.14,95.48)$ & 9.72 & $(-84.41,103.86)$ \\
\hline Age & 0.18 & $(0.08,0.27)$ & 0.17 & $(0.07,0.27)$ \\
\hline Years of education & -0.27 & $(-0.49,-0.06)$ & -0.27 & $(-0.50,-0.05)$ \\
\hline Male & 1.73 & $(0.60,2.87)$ & 1.78 & $(0.63,2.94)$ \\
\hline$>0$ APOE4 & 0.61 & $(-0.62,1.85)$ & 0.56 & $(-0.71,1.83)$ \\
\hline Amyloid status & -0.10 & $(-1.41,1.20)$ & -0.04 & $(-1.37,1.30)$ \\
\hline
\end{tabular}


Table 3 LME coefficients for longitudinal and cross-sectional models at 48 months from baseline

\begin{tabular}{|c|c|c|c|c|}
\hline & \multicolumn{2}{|l|}{ Longitudinal model } & \multicolumn{2}{|l|}{ Cross-sectional model } \\
\hline & Coefficient estimate & 95\% confidence interval & Coefficient estimate & 95\% confidence interval \\
\hline Participant ECog & 0.24 & $(-1.06,1.54)$ & 0.66 & $(-0.17,1.48)$ \\
\hline Study partner ECog & 1.18 & $(-0.39,2.75)$ & 1.37 & $(0.78,1.96)$ \\
\hline Time & -546.09 & $(-1469.04,376.87)$ & -526.34 & $(-1397.70,345.01)$ \\
\hline Squared time & 70.25 & $(-45.59,186.1)$ & 68.18 & $(-41.18,177.53)$ \\
\hline Age & 0.25 & $(0.05,0.45)$ & 0.21 & $(0.02,0.40)$ \\
\hline Years of education & -0.36 & $(-0.80,0.08)$ & -0.38 & $(-0.80,0.03)$ \\
\hline Male & 1.10 & $(-1.12,3.31)$ & 1.01 & $(-1.10,3.11)$ \\
\hline$>0$ APOE4 & 1.65 & $(-0.63,3.94)$ & 1.29 & $(-0.86,3.43)$ \\
\hline Amyloid status & 1.58 & $(-0.76,3.91)$ & 0.83 & $(-1.43,3.08)$ \\
\hline
\end{tabular}

performance. For example, a portion of MCI participants may demonstrate anosognosia, and these individuals may be at the highest risk for $\mathrm{AD}$ and progression to dementia [22, 23].

Notably, a minority $(N=47)$ of the participants in this study progressed to a clinical diagnosis of MCI. When removing those who converted to $\mathrm{MCI}$ at the 48-month time point, the observed cross-sectional effect disappeared. Partners' eMVI were no different from participants'. Thus, conversion to MCI is largely driving the observed overall effects at 48 months. Though this is important, it does not change the overall interpretation of our results. Indeed, preclinical AD trials aim to enrich for participants most likely to demonstrate cognitive decline during the course of study, ultimately including the onset of MCI or dementia in some participants. This is very much in line with the primary aim of these studies: to enable the efficient assessment of whether an intervention can delay or prevent the onset of cognitive impairment.

In contrast to our hypothesis, we did not observe differences in the associations between subjective reports and objective performance based on amyloid status. The length of the asymptomatic period of $\mathrm{AD}$ has been estimated to be as long as 15 years [24]. Recent demonstrations of the differences in cognitive performance between those with elevated and not elevated brain amyloid were most striking more than 48 months after baseline, beyond the length of longitudinal data used in the current study [25]. We chose this length of longitudinal assessment to recapitulate the lengths of preclinical AD trials, and also because of the limited data available based on our study design (i.e., the use of the ECog as a subjective assessment of participant cognitive performance). The risk for cognitive decline may also be highest in individuals who not only demonstrate elevated amyloid, but who are also carriers for the $\varepsilon 4$ allele of the APOE gene, and we lacked sufficient sample size to permit such further stratification in our analyses [26].

Amyloid status was positively associated with ADAS13 48 months from baseline but was not statistically significant due to high variation in the observed estimate. Predictors of ADAS13 scores (cross-sectionally and longitudinally) included age (positive association), education (negative association), and male sex (positive association). The effects of age and education are not surprising. The effect of sex, however, was unexpected, given that women are at increased risk for AD [27]. Men are, however, at increased risk for MCI and sex effects may yield differences not only in temporal progression of disease, but also specific cognitive domains that are affected early vs. late in disease [28, 29].

\section{Limitations}

As is the case with any observational study, there is a possibility that the relative informative ability of the study partners is affected by factors that were not observed. We attempted to mitigate this to the best of our ability by controlling for known confounding factors but were limited to the data related to the participants themselves. No data were available for the study partners. Characteristics such as whether the study partner lives with the participant or the number of hours spent per week in contact with the participant may affect the quality of data they provide [30], and an accompanying paper by our group examines this concept in preclinical $\mathrm{AD}$ trials [31]. In addition, due to protocol amendments in ADNI2, reliable data were not available at 36 months. We have no reason to suspect, however, that estimates would stray from the observed trajectories created by the 12-, 24-, and 48-month models. Moreover, there were few viable dyads remaining for 48-month analyses, limiting the estimate precision at this time point. More information at these time points may be necessary to credibly confirm the inferences made in this study.

\section{Conclusions}

While cognitively normal participants may be capable of providing consent and accurately informing on their own cognitive abilities at study start, study partner information is likely to become increasingly important over the course 
of a preclinical $\mathrm{AD}$ trial. Given that clinical trials tend to focus on current participant cognitive status measured prospectively in time, the finding that study partners provide increasingly more predictive data for assessing participant cognition as time moves on has potentially far-reaching implications in the setting of controlled intervention trials. Specifically, this finding suggests that the study partner role may be essential to minimizing bias, increasing precision in endpoint assessment, and ultimately ensuring trial data integrity. Thus, these results endorse the continued requirement of study partners in preclinical AD trials.

\begin{abstract}
Abbreviations
$A \beta_{42}$ : amyloid beta; AD: Alzheimer's disease; ADAS13: Alzheimer Disease Assessment Scale 13-item cognitive subscale; ADNI: Alzheimer's Disease Neuroimaging Initiative; ADNI2: Second phase of ADNI; CB: Confidence bound; Cl: Confidence interval; CSF: Cerebrospinal fluid; ECog: Everyday Cognition; eMVI: Estimated mean variable importance; FDA: Federal Drug Administration; LME: Linear mixed effects; MCl: Mild cognitive impairment; MRI: Magnetic resonance imaging; PET: Positron emission tomography; PiB: Pittsburgh compound B; SUVR: Standard uptake value ratio
\end{abstract}

\section{Acknowledgements}

Data used in the preparation of this paper were obtained from the Alzheimer's Disease Neuroimaging Initiative (ADNI) database ([30]). As such, the investigators within the ADNI contributed to the design and implementation of ADNI and/or provided data but did not participate in the analysis or in the writing of this paper. A complete listing of ADNI investigators can be found at http://adni.loni.usc.edu/wp-content/uploads/ how_to_apply/ADNI_Acknowledgement_List.pdf.

\section{Authors' contributions}

All authors participated in the conception, design, and analysis of the study. All authors also participated in the writing and editing of the manuscript, and all have read and approved the final manuscript.

\section{Funding}

This work was funded by NIA 1R21AG056931, AG016573, and AG059407. JDG is currently supported by UL1 TR000153. MMR is supported by NIA AG000096. Data collection and sharing for the Alzheimer's Disease Neuroimaging Initiative (ADNI) (National Institutes of Health Grant U01 AG024904) and DOD ADNI (Department of Defense award number W81XWH-12-2-0012). ADNI is funded by the National Institute on Aging, the National Institute of Biomedical Imaging and Bioengineering, and through generous contributions from the following: AbbVie, Alzheimer's Association; Alzheimer's Drug Discovery Foundation; Araclon Biotech; BioClinica, Inc;; Biogen; Bristol-Myers Squibb Company; CereSpir, Inc.; Cogstate; Eisai Inc.; Elan Pharmaceuticals, Inc.; Eli Lilly and Company; Eurolmmun; F. Hoffmann-La Roche Ltd. and its affiliated company Genentech, Inc.; Fujirebio; GE Healthcare; IXICO Ltd.; Janssen Alzheimer Immunotherapy Research \& Development, LLC.; Johnson \& Johnson Pharmaceutical Research \& Development LLC.; Lumosity; Lundbeck; Merck \& Co., Inc.; Meso Scale Diagnostics, LLC.; NeuroRx Research; Neurotrack Technologies; Novartis Pharmaceuticals Corporation; Pfizer Inc;; Piramal Imaging; Servier; Takeda Pharmaceutical Company; and Transition Therapeutics. The Canadian Institutes of Health Research is providing funds to support ADNI clinical sites in Canada. Private sector contributions are facilitated by the Foundation for the National Institutes of Health (www.fnih.org). The grantee organization is the Northern California Institute for Research and Education, and the study is coordinated by the Alzheimer's Therapeutic Research Institute at the University of Southern California. ADNI data are disseminated by the Laboratory for Neuro Imaging at the University of Southern California.

Availability of data and materials Not applicable.

\section{Ethics approval and consent to participate}

The study procedures were approved by the institutional review boards of all participating centers (https://adni.loni.usc.edu/wp-content/uploads/how_ to_apply/ADNI_Acknowledgement_List.pdf), and written informed consent was obtained from all participants or their authorized representatives. Ethics approval was obtained from the institutional review boards of each institution involved: Oregon Health and Science University; University of Southern California; University of California_-San Diego; University of Michigan; Mayo Clinic, Rochester; Baylor College of Medicine; Columbia University Medical Center; Washington University, St. Louis; University of Alabama at Birmingham; Mount Sinai School of Medicine; Rush University Medical Center; Wien Center; Johns Hopkins University; New York University; Duke University Medical Center; University of Pennsylvania; University of Kentucky; University of Pittsburgh; University of Rochester Medical Center; University of California, Irvine; University of Texas Southwestern Medical School; Emory University; University of Kansas, Medical Center; University of California, Los Angeles; Mayo Clinic, Jacksonville; Indiana University; Yale University School of Medicine; McGill University, Montreal-Jewish General Hospital; Sunnybrook Health Sciences, Ontario; U.B.C. Clinic for AD \& Related Disorders; Cognitive Neurology — St. Joseph's, Ontario; Cleveland Clinic Lou Ruvo Center for Brain Health; Northwestern University; Premiere Research Inst (Palm Beach Neurology); Georgetown University Medical Center; Brigham and Women's Hospital; Stanford University; Banner Sun Health Research Institute; Boston University; Howard University; Case Western Reserve University; University of California, Davis—Sacramento; Neurological Care of CNY; Parkwood Hospital; University of Wisconsin; University of California, Irvine—BIC; Banner Alzheimer's Institute; Dent Neurologic Institute; Ohio State University; Albany Medical College; Hartford Hospital, Olin Neuropsychiatry Research Center; Dartmouth-Hitchcock Medical Center; Wake Forest University Health Sciences; Rhode Island Hospital; Butler Hospital; UC San Francisco; Medical University South Carolina; St. Joseph's Health Care Nathan Kline Institute; University of lowa College of Medicine; and Cornell University and University of South Florida: USF Health Byrd Alzheimer's Institute. The investigators within the ADNI contributed to the design and implementation of the ADNI and/or provided data but did not participate in the analysis or writing of this report. A complete listing of ADNI investigators can be found online (http://adni. loni.usc.edu/wp-content/uploads/how_to_apply/ADNI_Acknowledgement_ List.pdf).

\section{Consent for publication}

Not applicable.

\section{Competing interests}

The authors declare that they have no competing interests.

\section{Author details}

'Department of Statistics, University of California, Irvine, Irvine, CA 92697, USA. ${ }^{2}$ Institute for Memory Impairments and Neurological Disorders, University of California, Irvine 92697, CA, USA. ${ }^{3}$ Department of Psychiatry and Human Behavior, University of California, Irvine, Irvine, CA 92697, USA. ${ }^{4}$ Department of Neurobiology and Behavior, University of California, Irvine, Irvine, CA 92697, USA. ${ }^{5}$ Institute for Clinical and Translational Science, University of California, Irvine, Irvine, CA 92697, USA.

Received: 24 May 2019 Accepted: 21 September 2019

Published online: 18 October 2019

\section{References}

1. U.S. Department of Health and Human Services, Food and Drug Administration, Center for Drug Evaluation and Research (CDER), Center for Biologics Evaluation and Research (CBER). Early Alzheimer's disease: developing drugs for treatment guidance for industry - draft guidance; 2018. p. 10.

2. Largent EA, Karlawish J, Grill JD. Study partners: essential collaborators in discovering treatments for Alzheimer's disease. Alzheimers Res Ther. 2018; 10:101. https://doi.org/10.1186/s13195-018-0425-4 [cited 2018 Oct 24].

3. Hardy JA, Higgins GA. Alzheimer's disease: the amyloid cascade hypothesis. Science. 1992;256:184-5 Available from: http://science.sciencemag.org/ content/256/5054/184. [cited 2019 Feb 19].

4. Albert MS, DeKosky ST, Dickson D, Dubois B, Feldman HH, Fox NC, et al. The diagnosis of mild cognitive impairment due to Alzheimer's disease: 
recommendations from the National Institute on Aging-Alzheimer's Association workgroups on diagnostic guidelines for Alzheimer's disease. Alzheimers Dement. 2011;7:270-9 Available from: http://www.sciencedirect. com/science/article/pii/S155252601100104X. [cited 2019 Mar 22].

5. Dubois B, Feldman HH, Jacova C, Cummings JL, Dekosky ST, BarbergerGateau P, et al. Revising the definition of Alzheimer's disease: a new lexicon. Lancet Neurol. 2010;9:1118-27 Available from: http://www.sciencedirect. com/science/article/pii/S1474442210702234. [cited 2019 Mar 22].

6. Sperling RA, Aisen PS, Beckett LA, Bennett DA, Craft S, Fagan AM, et al. Toward defining the preclinical stages of Alzheimer's disease: recommendations from the National Institute on Aging-Alzheimer's Association workgroups on diagnostic guidelines for Alzheimer's disease. Alzheimers Dement. 2011;7:280-92 Available from: http://www. sciencedirect.com/science/article/pii/S1552526011000999. [cited 2019 Feb 27]:

7. Tierney MC, Szalai JP, Snow WG, Fisher RH. The prediction of Alzheimer disease: the role of patient and informant perceptions of cognitive deficits. Arch Neurol. 1996;53:423-7 Available from: https://jamanetwork.com/ journals/jamaneurology/fullarticle/594012. [cited 2019 Mar 22].

8. Tierney MC, Herrmann N, Geslani DM, Szalai JP. Contribution of informant and patient ratings to the accuracy of the Mini-Mental State Examination in predicting probable Alzheimer's disease. J Am Geriatr Soc. 2003;51:813-8 Available from: https://onlinelibrary.wiley.com/doi/abs/10.1046/j.1365-2389.2 003.51262.x. [cited 2019 Mar 22].

9. Piras F, Piras F, Orfei MD, Caltagirone C, Spalletta G. Self-awareness in mild cognitive impairment: quantitative evidence from systematic review and metaanalysis. Neurosci Biobehav Rev. 2016;61:90-107 Available from: http://www. sciencedirect.com/science/article/pii/S0149763415002626. [cited 2019 Jul 25].

10. Farias ST, Lau K, Harvey D, Denny KG, Barba C, Mefford AN. Early functional limitations in cognitively normal older adults predict diagnostic conversion to mild cognitive impairment. J Am Geriatr Soc. 2017;65:1152-8 Available from: https:/onlinelibrary.wiley.com/doi/abs/10.1111/jgs.14835. [cited 2018 Oct 1].

11. Amariglio RE, Donohue MC, Marshall GA, Rentz DM, Salmon DP, Ferris SH, et al. Tracking early decline in cognitive function in older individuals at risk for Alzheimer disease dementia: the Alzheimer's Disease Cooperative Study Cognitive Function Instrument. JAMA Neurol. 2015;72:446-54 Available from: https://jamanetwork.com/journals/jamaneurology/fullarticle/2110225. [cited 2018 Oct 1].

12. Grill JD, Karlawish J. Study partners should be required in preclinical Alzheimer's disease trials. Alzheimers Res Ther. 2017;9:93. https://doi.org/10. 1186/s13195-017-0327-x [cited 2018 Dec 3].

13. Albert M, DeKosky S, Salmon D, Morris J, Cairns N. Alzheimer's disease neuroimaging initiative 2 (adni2) protocol (ADC-039). 2015;59.

14. Landau SM, Breault $C$, Joshi AD, Pontecorvo M, Mathis CA, Jagust WJ, et al. Amyloid- $B$ imaging with Pittsburgh compound $B$ and florbetapir: comparing radiotracers and quantification methods. J Nucl Med. 2013;54:70-7 Available from: http://jnm.snmjournals.org/content/54/1/70. [cited 2019 Feb 22].

15. Mohs RC, Knopman D, Petersen RC, Ferris SH, Ernesto C, Grundman M, et al. Development of cognitive instruments for use in clinical trials of antidementia drugs: additions to the Alzheimer's Disease Assessment Scale that broaden its scope. The Alzheimer's Disease Cooperative Study. Alzheimer Dis Assoc Disord. 1997;11(Suppl 2):S13-21.

16. Sano M, Raman R, Emond J, Thomas RG, Petersen R, Schneider LS, et al. Adding delayed recall to the Alzheimer Disease Assessment Scale is useful in studies of mild cognitive impairment but not Alzheimer disease. Alzheimer Dis Assoc Disord. 2011;25:122 Available from: https://journals.lww. com/alzheimerjournal/fulltext/2011/04000/Adding_Delayed_Recall_to_the_ Alzheimer_Disease.4.aspx. [cited 2019 Mar 22].

17. Farias ST, Mungas D, Reed BR, Cahn-Weiner D, Jagust W, Baynes K, et al. The measurement of Everyday Cognition (ECog): scale development and psychometric properties. Neuropsychology. 2008;22:531-44 Available from: https://www.ncbi.nlm.nih.gov/pmc/articles/PMC2877034/. [cited 2018 Oct 1].

18. Breiman L. Random forests. Mach Learn. 2001;45:5-32. https://doi.org/10. 1023/A:1010933404324 [cited 2019 Feb 27].

19. R Core Team. R: a language and environment for statistical computing. Vienna: R Foundation for statistical Computing; 2017. Available from: https:// www.R-project.org/

20. Julie Tibshirani, Susan Athey, Stefan Wager, Rina Friedberg, Luke Miner, Marvin Wright. grf: generalized random forests (beta) 2018. Available from: https://CRAN.R-project.org/package=grf
21. Follmann D, Proschan M, Leifer E. Multiple outputation: inference for complex clustered data by averaging analyses from independent data. Biometrics. 2003;59:420-9 Available from: https://onlinelibrary.wiley.com/doi/ abs/10.1111/1541-0420.00049. [cited 2018 Nov 18].

22. Munro CE, Donovan NJ, Amariglio RE, Papp KV, Marshall GA, Rentz DM, et al. The impact of awareness of and concern about memory performance on the prediction of progression from mild cognitive impairment to Alzheimer disease dementia. Am J Geriatr Psychiatry. 2018;26:896-904 Available from: http://www.sciencedirect.com/science/article/pii/S1064748118303233. [cited 2019 Mar 25].

23. Therriault J, Ng KP, Pascoal TA, Mathotaarachchi S, Kang MS, Struyfs H, et al. Anosognosia predicts default mode network hypometabolism and clinical progression to dementia. Neurology. 2018;90:e932-9 Available from: http:// www.neurology.org/lookup/doi/10.1212/WNL.0000000000005120. [cited 2019 Mar 25].

24. Sperling R, Mormino E, Johnson K. The evolution of preclinical Alzheimer's disease: implications for prevention trials. Neuron. 2014;84:608-22 Available from: http://www.sciencedirect.com/science/article/pii/S0896627314009581. [cited 2019 Mar 25].

25. Donohue MC, Sperling RA, Petersen R, Sun C-K, Weiner MW, Aisen PS. Association between elevated brain amyloid and subsequent cognitive decline among cognitively normal persons. JAMA. 2017;317:2305-16 Available from: https://jamanetwork.com/journals/jama/fullarticle/2631529. [cited 2018 Oct 1].

26. Lim YY, Kalinowski P, Pietrzak RH, Laws SM, Burnham SC, Ames D, et al. Association of $\beta$-amyloid and apolipoprotein $E \varepsilon 4$ with memory decline in preclinical Alzheimer disease. JAMA Neurol. 2018;75:488-94 Available from: https://jamanetwork.com/journals/jamaneurology/fullarticle/2670442. [cited 2019 Mar 25].

27. Mielke MM, Vemuri P, Rocca WA. Clinical epidemiology of Alzheimer's disease: assessing sex and gender differences. Clin Epidemiol. 2014;6:37-48 Available from: https://www.ncbi.nlm.nih.gov/pmc/articles/PMC3891487/. [cited 2019 Mar 25]

28. Petersen RC, Roberts RO, Knopman DS, Geda YE, Cha RH, Pankratz VS, et al. Prevalence of mild cognitive impairment is higher in men: the Mayo Clinic Study of Aging. Neurology. 2010;75:889-97 Available from: http://www. neurology.org/cgi/doi/10.1212/WNL.0b013e3181f11d85. [cited 2019 Mar 25].

29. Sundermann EE, Biegon A, Rubin LH, Lipton RB, Mowrey W, Landau S, et al. Better verbal memory in women than men in $\mathrm{MCl}$ despite similar levels of hippocampal atrophy. Neurology. 2016;86:1368-76 Available from: http:// www.neurology.org/lookup/doi/10.1212/WNL.0000000000002570. [cited 2019 Mar 25]

30. Ready R, Ott B, Grace J. Validity of informant reports about $A D$ and $M C l$ patients' memory. Alzheimer Dis Assoc Disord. 2004;18:11-6 Available from: insights.ovid.com. [cited 2019 Mar 27].

31. Nuño MM, Gillen DL, Grill JD. Study Partner Types and Prediction of Cognitive Performance: Implications to Pre-Clinical Alzheimer's Trials. Alzheimers Res Ther. In press

\section{Publisher's Note}

Springer Nature remains neutral with regard to jurisdictional claims in published maps and institutional affiliations.

Ready to submit your research? Choose BMC and benefit from:

- fast, convenient online submission

- thorough peer review by experienced researchers in your field

- rapid publication on acceptance

- support for research data, including large and complex data types

- gold Open Access which fosters wider collaboration and increased citations

- maximum visibility for your research: over $100 \mathrm{M}$ website views per year

At $\mathrm{BMC}$, research is always in progress.

Learn more biomedcentral.com/submission 